\title{
New Supersymmetric Contributions to Neutrinoless Double Beta Decay
}

\author{
M. Hirsch ${ }^{1}$, H.V. Klapdor-Kleingrothaus ${ }^{2}$ \\ Max-Plank-Institut für Kernphysik, P.O. 1039 80, D-69029, Heidelberg, \\ Germany \\ S.G. Kovalenkoß \\ Joint Institute for Nuclear Research, Dubna, Russia
}

\begin{abstract}
The neutrinoless double beta $(0 \nu \beta \beta)$ decay is analyzed within the Minimal Supersymmetric Standard Model with explicit R-parity violation $\left(\not R_{p} \mathrm{MSSM}\right)$. We have found new supersymmetric contributions to this process and give the complete set of relevant Feynman diagrams. Operators describing $0^{+} \longrightarrow 0^{+}$nuclear transitions induced by the supersymmetric interactions of the $\not R_{p} \mathrm{MSSM}$ are derived. These operators can be used for calculating the $0 \nu \beta \beta$ decay rate applying any specific nuclear model wave functions.
\end{abstract}

The observation of neutrinoless double beta $(0 \nu \beta \beta)$ decay would be a clear signal for physics beyond the standard model, since it violates lepton number by two units (for reviews see [1], [2]). No definite observation of $0 \nu \beta \beta$ decay has been reported to date, but recent experimental progress has pushed the existing half-life limits of $0 \nu \beta \beta$ decay beyond $10^{24}$ years and further progress can be expected in the near future [3].

Half-life limits on $0 \nu \beta \beta$ decay are usually interpreted as limits on the effective Majorana neutrino mass (see fig. 1). However, it is known for some time that there exist also other mechanisms which might induce $0 \nu \beta \beta$ decays, like for example in left-right symmetric extensions of the standard model gauge group [2]. In this paper we study contributions to $0 \nu \beta \beta$ decay within supersymmetric (SUSY) theories with explicit R-parity breaking. R-parity $\left(R_{p}\right)$ is a discrete, multiplicative symmetry defined as $R_{p}=(-1)^{3 B+L+2 S}$, where $S, B$

\footnotetext{
${ }^{1}$ MAHIRSCH@ENULL.MPI-HD.MPG.DE

${ }^{2}$ KLAPDOR@ENULL.MPI-HD.MPG.DE

${ }^{3}$ KOVALEN@NUSUN.JINR.DUBNA.SU
} 
and $L$ are the spin, the baryon and the lepton quantum number. Conservation of R-parity in turn implies baryon number $(B)$ and lepton number $(L)$ conservation. This symmetry has been imposed on the minimal supersymmetric standard model (MSSM) (for a review see [4]). However, neither gauge invariance nor supersymmetry require $R_{p}$ conservation. The question whether or not $R_{p}$ is a good symmetry of the supersymmetric theory is a dynamical problem which might be related to more fundamental physics at the Planck scale. In general, $R_{p}$ can be either broken explicitly [5], [6] or spontaneously [7], [8] by the vacuum expectation value of the scalar superpartner of the $R_{p}$-odd isosinglet lepton field [8]. Supersymmetric models with $R_{p}$ non-conservation $\left(R_{p}\right)$ have been extensively discussed in the literature not only because of their great theoretical interest, but also because they have interesting phenomenological [9], [10], [11], and cosmological [12]-[15] implications. One can expect that $\not R_{p}$ SUSY models give a very natural framework for lepton number violating processes and particularly $0 \nu \beta \beta$ decay [20].

Let us start with a brief review of the model we use in the present paper.

We follow the MSSM extended by inclusion of explicit $R$-parity violating $\left(\not R_{p}\right)$ terms into the superpotential. This model $\left(\not R_{p} \mathrm{MSSM}\right)$ has the MSSM field content and is completely specified by the standard $S U(3) \times S U(2) \times U(1)$ gauge couplings as well as by the low-energy superpotential and "soft" SUSY breaking terms [4]. The most general gauge invariant form of the superpotential is [11, 16]

$$
W=W_{R_{p}}+W_{\not R_{p}} \text {. }
$$

The $R_{p}$ conserving part has the standard MSSM form

$$
W_{R_{p}}=h_{L} H_{1} L \bar{E}+h_{D} H_{1} Q \bar{D}-h_{U} H_{2} Q \bar{U}-\mu H_{1} H_{2} \text {. }
$$

We use notations $L, Q$ for lepton and quark doublet superfields and $\bar{E}, \bar{U}, \bar{D}$ for lepton and up, down quark singlet superfields; $H_{1}$ and $H_{2}$ are the Higgs doublet superfields with a weak hypercharge $Y=-1,+1$, respectively. Summation over generations is implied. For simplicity generation indices of fields and Yukawa coupling constants $h_{L}, h_{U}, h_{D}$ are suppressed. The mass-mixing parameter $\mu$ is a free parameter describing mixing between the Higgs bosons $H_{1}-H_{2}$ as well as between higgsinos $\tilde{H}_{1}-\tilde{H}_{2}$.

The $R_{p}$ violating part of the superpotential (1) can be written as [5], [6],

$$
W_{\mathbb{R}_{p}}=\lambda_{i j k} L_{i} L_{j} \bar{E}_{k}+\lambda_{i j k}^{\prime} L_{i} Q_{j} \bar{D}_{k}+\lambda_{i j k}^{\prime \prime} \bar{U}_{i} \bar{D}_{j} \bar{D}_{k}
$$

where indices $i, j, k$ denote generations, and the fields have been defined so that the bilinear lepton number violating operators $L_{i} H_{2}$ [6 have been rotated 
away. The coupling constants $\lambda\left(\lambda^{\prime \prime}\right)$ are antisymmetric in the first (last) two indices. The first two terms lead to lepton number violation, while the last one violates baryon number conservation. Proton stability forbids simultaneous presence of lepton and baryon number violating terms in the superpotential [10] (unless the couplings are very small). Therefore, only $\lambda, \lambda^{\prime}$ or $\lambda^{\prime \prime}$ type interactions can be present. There may exist an underlying discrete symmetry in the theory which allows either the first or the second set of couplings. This discrete symmetry, called matter parity [11], [16] can be imposed 'ad hoc' on the MSSM. It can be justified on a more fundamental level of Planck scale physics. Such a discrete symmetry is shown to be compatible with the ordinary $S U(5)$ [6] and "flipped" $S U(5) \times U(1)$ [17] grand unification (GUT) scenarios, as well as with phenomenologically viable superstring theories [18].

Neutrinoless double beta decay, which is the main subject of the present paper, requires lepton number violating interactions. Therefore we bind ourselves to the $\not R_{p}$ MSSM with lepton number violation $\left(\lambda \neq 0, \lambda^{\prime} \neq 0\right)$ and baryon number conservation $\left(\lambda^{\prime \prime}=0\right)$. Apparently, $0 \nu \beta \beta$ can probe only the first generation lepton number violating Yukawa coupling $\lambda_{111}^{\prime}$ because only the first generation fermions $u, d, e$ are involved in this process.

In addition to proton decay constraints on $\not R_{p}$ couplings there are also constraints which follow from cosmological arguments requiring that the baryon asymmetry generated at the GUT scale is not washed out by $B-L$ violating interactions present in eq. (3). These cosmological constraints have been thought to affect all $\not R_{p}$ couplings $\lambda, \lambda^{\prime}, \lambda^{\prime \prime} \ll 10^{-7}$, making these models phenomenologically not interesting. These arguments, however, were proved to be strongly model dependent [14], [15]. Moreover it was shown recently that the cosmological bounds can be evaded in perfectly reasonable scenarios of matter genesis [15].

The effect of "soft" supersymmetry breaking can be parametrized at the Fermi scale as a part of the scalar potential:

$$
\begin{array}{r}
V_{\text {soft }}=\sum_{i=\text { scalars }} m_{i}^{2}\left|\phi_{i}\right|^{2}+h_{L} A_{L} H_{1} \tilde{L} \tilde{\bar{E}}+h_{D} A_{D} H_{1} \tilde{Q} \tilde{\bar{D}}-h_{U} A_{U} H_{2} \tilde{Q} \tilde{\bar{U}}- \\
-\mu B H_{1} H_{2}+\text { h.c. }
\end{array}
$$

and a "soft" gaugino mass term

$$
\mathcal{L}_{G M}=-\frac{1}{2}\left[M_{1} \tilde{B} \tilde{B}+M_{2} \tilde{W}^{k} \tilde{W}^{k}+M_{3} \tilde{g}^{a} \tilde{g}^{a}\right]-\text { h.c. }
$$


As usual, $M_{3,2,1}$ are the masses of the $S U(3) \times S U(2) \times U(1)$ gauginos $\tilde{g}, \tilde{W}, \tilde{B}$ and $m_{i}$ are the masses of scalar fields. $A_{L}, A_{D}, A_{U}$ and $B$ are trilinear and bilinear "soft" supersymmetry breaking parameters. Here, $m_{\tilde{g}}=M_{3}$ stands for the gluino $\tilde{g}$ mass.

Now the model is completely specified and we can deduce the interaction terms of the $\not R_{p}$ MSSM - Lagrangian relevant for neutrinoless double beta decay.

Let us write down these interaction terms explicitly. In the following we use the 4-component Dirac bispinor notation for fermion fields.

The lepton number violating part of the Lagrangian can be obtained directly from the $W_{\mathbb{R}_{p}}$ superpotential part (3). It has the form

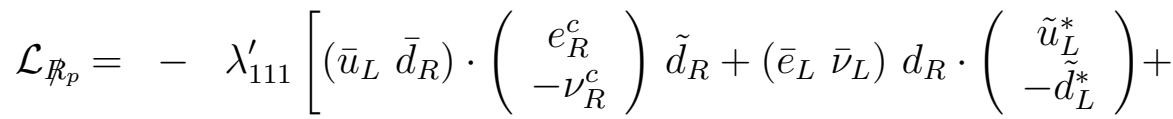

$$
\begin{aligned}
& \left.+\left(\bar{u}_{L} \bar{d}_{L}\right) d_{R} \cdot\left(\begin{array}{c}
\tilde{e}_{L}^{*} \\
-\tilde{\nu}_{L}^{*}
\end{array}\right)+\text { h.c. }\right]
\end{aligned}
$$

To construct diagrams contributing to $0 \nu \beta \beta$ decay we will also need $R_{p}$ conserving gaugino-fermion-sfermion vertices.

The Lagrangian terms corresponding to gluino $\mathcal{L}_{\tilde{g}}$ and neutralinos $\mathcal{L}_{\chi}$ interactions with fermions $\psi=\{u, d, e\}, q=\{u, d\}$ and their superpartners $\tilde{\psi}=\{\tilde{u}, \tilde{d}, \tilde{e}\}, \tilde{q}=\{\tilde{u}, \tilde{d}\}$ are $[$ 田

$$
\begin{gathered}
\mathcal{L}_{\tilde{g}}=-\sqrt{2} g_{3} \frac{\lambda_{\alpha \beta}^{(a)}}{2}\left(\bar{q}_{L}^{\alpha} \tilde{g} \tilde{q}_{L}^{\beta}-\bar{q}_{R}^{\alpha} \tilde{g} \tilde{q}_{R}^{\beta}\right)+\text { h.c. } \\
\mathcal{L}_{\chi}=\sqrt{2} g_{2} \sum_{i=1}^{4}\left(\epsilon_{L i}(\psi) \bar{\psi}_{L} \chi_{i} \tilde{\psi}_{L}+\epsilon_{R i}(\psi) \bar{\psi}_{R} \chi_{i} \tilde{\psi}_{R}\right)+\text { h.c. }
\end{gathered}
$$

Here $\lambda^{(a)}$ are $3 \times 3$ Gell-Mann matrices $(a=1, \ldots, 8)$. Neutralino coupling constants are defined as [4]

$$
\begin{aligned}
\epsilon_{L i}(\psi) & =-T_{3}(\psi) \mathcal{N}_{i 2}+\tan \theta_{W}\left(T_{3}(\psi)-Q(\psi)\right) \mathcal{N}_{i 1} \\
\epsilon_{R i}(\psi) & =Q(\psi) \tan \theta_{W} \mathcal{N}_{i 1} .
\end{aligned}
$$

Here $Q(\psi)$ and $T_{3}(\psi)$ are the electric charge and weak isospin of the field $\psi$.

The coefficients $N_{i j}$ are elements of the orthogonal mixing matrix which diagonalizes the $4 \times 4$ neutralino mass matrix [4]. The four neutralino mass 
eigenstates $\chi_{i}$ with masses $m_{\chi_{i}}{ }^{1}$ have the field content

$$
\chi_{i}=\mathcal{N}_{i 1} \tilde{B}+\mathcal{N}_{i 2} \tilde{W}^{3}+\mathcal{N}_{i 3} \tilde{H}_{1}^{0}+\mathcal{N}_{i 4} \tilde{H}_{2}^{0}
$$

Recall again that we use notations $\tilde{W}^{3}, \tilde{B}$ for neutral $S U(2)_{L} \times U(1)$ gauginos and $\tilde{H}_{2}^{0}, \tilde{H}_{1}^{0}$ for higgsinos which are the superpartners of the two neutral Higgs boson fields $H_{1}^{0}$ and $H_{2}^{0}$.

Having specified the Lagrangian interaction terms (6) - (8) one can construct diagrams describing the $\not R_{p} \mathrm{MSSM}$ contribution to the $0 \nu \beta \beta$ decay. The complete set of these diagrams is presented in fig. 2 .

The supersymmetric mechanism of $0 \nu \beta \beta$ decay was first proposed by Mohapatra [20] and later studied in more details by Vergados [21]. In these papers [20], 21] only three diagrams similar to those in Fig.2(a) were considered. Instead of neutralinos $\chi_{i}$, which are actual mass eigenstates in the MSSM, the consideration of refs. [20], [21] used Z-ino $(\tilde{Z})$ and photino $(\tilde{\gamma})$ fields in intermediate states. $\tilde{Z}$ and $\tilde{\gamma}$ can be mass eigenstates only at special values of parameters of the neutralino mass matrix. In general, these fields are not mass eigenstates. Using such fields in the intermediate states leads to neglecting diagrams with mixed intermediate states when, for instance, $\tilde{Z}$ turns to $\tilde{\gamma}$ due to the mixing proportional to the relevant entry of the neutralino mass matrix. The effect of mixing is taken into account completely in the diagrams displayed in fig.2(a,b) with all neutralino mass eigenstates $\chi_{i}$ involved. Note that diagrams in fig. 2(b) were previously not considered in the literature.

In the case of $0 \nu \beta \beta$ decay when momenta of external particles are much smaller then intermediate particle masses one can treat the interactions in Figs 1,2 as point-like. A suitable formalism in this case is the effective Lagrangian approach.

It is now straightforward to find the operators in the effective Lagrangian which correspond to the diagrams in Fig. 2(a,b). The result is

$$
\begin{aligned}
& \mathcal{L}_{\text {eff }}^{\Delta L_{e}=2}(x)=8 \pi \alpha_{2} \lambda_{111}^{\prime 2} \sum_{i=1}^{4} \frac{1}{m_{\chi_{i}}}\left[\frac{\epsilon_{L i}^{2}(e)}{m_{\tilde{e}_{L}}^{4}}\left(\bar{u}_{L}^{\alpha} d_{R \alpha}\right)\left(\bar{u}_{L}^{\beta} d_{R \beta}\right)\left(\bar{e}_{L} e_{R}^{\mathbf{c}}\right)+\right. \\
& +\frac{\epsilon_{L i}^{2}(u)}{m_{\tilde{u}_{L}}^{4}}\left(\bar{u}_{L}^{\alpha} u_{R \beta}^{\mathbf{c}}\right)\left(\bar{e}_{L} d_{R \alpha}\right)\left(\bar{e}_{L} d_{R}^{\beta}\right)+\frac{\epsilon_{R i}^{2}(d)}{m_{\tilde{d}_{R}}^{4}}\left(\bar{u}_{L}^{\alpha} e_{R}^{\mathbf{c}}\right)\left(\bar{u}_{L}^{\beta} e_{R}^{\mathbf{c}}\right)\left({\overline{d^{\mathbf{c}}}}_{L \alpha} d_{R \beta}\right)+
\end{aligned}
$$

\footnotetext{
${ }^{1}$ The neutralino masses $m_{\chi_{i}}$ can be either positive or negative if the orthogonal matrix $N$ is used in the diagonalization procedure [19]. The sign of the mass coincides with the $\mathrm{CP}$-parity of the corresponding neutralinos $\chi_{i}$.
} 


$$
\begin{aligned}
& \left.+\left(\frac{\epsilon_{L i}(u) \epsilon_{R i}(d)}{m_{\tilde{u}_{L}}^{2} m_{\tilde{d}_{R}}^{2}}+\frac{\epsilon_{L i}(u) \epsilon_{L i}(e)}{m_{\tilde{u}_{L}}^{2} m_{\tilde{e}_{L}}^{2}}+\frac{\epsilon_{L i}(e) \epsilon_{R i}(d)}{m_{\tilde{e}_{L}}^{2} m_{\tilde{d}_{R}}^{2}}\right)\left(\bar{u}_{L}^{\alpha} d_{R}^{\beta}\right)\left(\bar{u}_{L \beta} e_{R}^{\mathbf{c}}\right)\left(\bar{e}_{L} d_{R \alpha}\right)\right]+ \\
& +\lambda_{111}^{\prime 2} \frac{8 \pi \alpha_{s}}{m_{\tilde{g}}} \frac{\lambda_{\alpha \beta}^{(a)}}{2} \frac{\lambda_{\gamma \delta}^{(a)}}{2}\left[\frac{1}{m_{\tilde{u}_{L}}^{4}}\left(\bar{u}_{L}^{\alpha} u_{R}^{\mathbf{c} \gamma}\right)\left(\bar{e}_{L} d_{R}^{\beta}\right)\left(\bar{e}_{L} d_{R}^{\delta}\right)+\right. \\
& \left.+\frac{1}{m_{\tilde{d}_{R}}^{4}}\left(\bar{u}_{L}^{\alpha} e_{R}^{\mathbf{c}}\right)\left(\bar{u}_{L}^{\gamma} e_{R}^{\mathbf{c}}\right)\left(\bar{d}_{L}^{\mathbf{c}}{ }_{L}^{\beta} d_{R}^{\mathbf{c}}{ }^{\delta}\right)-\frac{1}{m_{\tilde{d}_{R}}^{2} m_{\tilde{u}_{L}}^{2}}\left(\bar{u}_{L}^{\alpha} d_{R}^{\delta}\right)\left(\bar{u}_{L}^{\gamma} e_{R}^{\mathbf{c}}\right)\left(\bar{e}_{L} d_{R}^{\beta}\right)\right]
\end{aligned}
$$

Here $\alpha_{2}=g_{2}^{2} /(4 \pi)$ and $\alpha_{s}=g_{3}^{2} /(4 \pi)$.

The Lagrangian (11) has terms in a form which do not allow a direct application of the non-relativistic impulse approximation which is necessary for the standard calculation of the $0 \nu \beta \beta$ reaction matrix element [1], [2]. One should rearrange the right hand side of eq. (11) in the form of a product of two colour-singlet quark currents and the leptonic current. It can be accomplished by a Fierz rearrangement procedure and subsequent extraction of color-singlets from the product of two colour-triplet and colour-antitriplet quark fields. The final result is

$$
\begin{aligned}
\mathcal{L}_{e f f}^{\Delta L_{e}=2}(x) & =\frac{G_{F}^{2}}{2} \cdot m_{P}^{-1}\left[\left(\eta_{\tilde{g}}+\eta_{\chi}\right)\left(J_{P S} J_{P S}-\frac{1}{4} J_{T}^{\mu \nu} J_{T \mu \nu}\right)+\right. \\
& \left.+\left(\eta_{\chi \tilde{e}}+\eta_{\tilde{g}}^{\prime}-\eta_{\chi \tilde{f}}\right) J_{P S} J_{P S}+\eta_{N} J_{V A}^{\mu} J_{V A \mu}\right]\left(\bar{e}\left(1+\gamma_{5}\right) e^{\mathbf{c}}\right) .
\end{aligned}
$$

The last term, corresponding to the heavy Majorana neutrino exchange diagram in fig.1, is included for completeness. The lepton number violating parameters are defined as follows

$$
\begin{aligned}
\eta_{\tilde{g}} & =\frac{2 \pi \alpha_{s}}{9} \frac{\lambda_{111}^{\prime 2}}{G_{F}^{2} m_{\tilde{d}_{R}}^{4}} \frac{m_{P}}{m_{\tilde{g}}}\left[1+\left(\frac{m_{\tilde{d}_{R}}}{m_{\tilde{u}_{L}}}\right)^{4}\right] \\
\eta_{\chi} & =\frac{\pi \alpha_{2}}{6} \frac{\lambda_{111}^{\prime 2}}{G_{F}^{2} m_{\tilde{d}_{R}}^{4}} \sum_{i=1}^{4} \frac{m_{P}}{m_{\chi_{i}}}\left[\epsilon_{R i}^{2}(d)+\epsilon_{L i}^{2}(u)\left(\frac{m_{\tilde{d}_{R}}}{m_{\tilde{u}_{L}}}\right)^{4}\right] \\
\eta_{\chi \tilde{e}} & =2 \pi \alpha_{2} \frac{\lambda_{111}^{\prime 2}}{G_{F}^{2} m_{\tilde{d}_{R}}^{4}}\left(\frac{m_{\tilde{d}_{R}}}{m_{\tilde{e}_{L}}}\right)^{4} \sum_{i=1}^{4} \epsilon_{L i}^{2}(e) \frac{m_{P}}{m_{\chi_{i}}}, \\
\eta_{\tilde{g}}^{\prime} & =\frac{4 \pi \alpha_{s}}{9} \frac{\lambda_{111}^{\prime 2}}{G_{F}^{2} m_{\tilde{d}_{R}}^{4}} \frac{m_{P}}{m_{\tilde{g}}}\left(\frac{m_{\tilde{d}_{R}}}{m_{\tilde{u}_{L}}}\right)^{2}, \\
\eta_{\chi \tilde{f}} & =\frac{\pi \alpha_{2}}{3} \frac{\lambda_{111}^{\prime 2}}{G_{F}^{2} m_{\tilde{d}_{R}}^{4}}\left(\frac{m_{\tilde{d}_{R}}}{m_{\tilde{e}_{L}}}\right)^{2} \sum_{i=1}^{4} \frac{m_{P}}{m_{\chi_{i}}}\left[\epsilon_{R i}(d) \epsilon_{L i}(e)+\right.
\end{aligned}
$$




$$
\begin{aligned}
& \left.+\epsilon_{L i}(u) \epsilon_{R i}(d)\left(\frac{m_{\tilde{e}_{L}}}{m_{\tilde{u}_{L}}}\right)^{2}+\epsilon_{L i}(u) \epsilon_{L i}(e)\left(\frac{m_{\tilde{d}_{R}}}{m_{\tilde{u}_{L}}}\right)^{2}\right], \\
\eta_{N} & =\frac{m_{P}}{<m_{N}>},
\end{aligned}
$$

where $<m_{N}>$ is the effective heavy Majorana neutrino mass (for definition see [2]).

Colour-singlet hadronic currents have the form

$$
\begin{aligned}
J_{P S} & =\bar{u}^{\alpha}\left(1+\gamma_{5}\right) d_{\alpha}, J_{T}^{\mu \nu}=\bar{u}^{\alpha} \sigma^{\mu \nu}\left(1+\gamma_{5}\right) d_{\alpha}, \\
J_{A V}^{\mu} & =\bar{u}^{\alpha} \gamma^{\mu}\left(1-\gamma_{5}\right) d_{\alpha},
\end{aligned}
$$

where $\sigma^{\mu \nu}=\frac{i}{2}\left[\gamma^{\mu}, \gamma^{\nu}\right]$.

Let us write down the $0 \nu \beta \beta$ decay matrix element $\mathcal{R}_{0 \nu \beta \beta}$ corresponding to the effective Lagrangian eq. (12)

$$
\begin{aligned}
\mathcal{R}_{0 \nu \beta \beta}= & \frac{G_{F}^{2}}{\sqrt{2}} m_{P}^{-1} C_{0 \nu}^{-1}\left\{\bar{e}\left(1+\gamma_{5}\right) e^{\mathbf{c}}\right\} \times \\
& {\left[\left(\eta_{\tilde{g}}+\eta_{\chi}\right)\left\langle F\left|\Omega_{\tilde{q}}\right| I\right\rangle+\left(\eta_{\chi \tilde{e}}+\eta_{\tilde{g}}^{\prime}-\eta_{\chi \tilde{f}}\right)\left\langle F\left|\Omega_{\tilde{f}}\right| I\right\rangle+\eta_{N}\left\langle F\left|\Omega_{N}\right| I\right\rangle\right] . }
\end{aligned}
$$

The heavy Majorana neutrino exchange contribution has been included in the last term of this equation. It corresponds to the last term of eq. (12). The normalization factor $C_{0 \nu}$ is defined below.

We have introduced transition operators $\Omega_{i}$ (for definitions see [2]) which are useful for separating the particle physics part of the calculation from the nuclear physics one. The transition operators contain information about the underlying interactions at the quark level (12) and quark states inside the nucleon. They are independent of the initial $\mid I>$ and the final $<F \mid$ nuclear states. To calculate the nuclear matrix elements in eq. (21) one may use any nuclear model wave functions. In our case $\Omega_{\tilde{q}}, \Omega_{\tilde{f}}$ and $\Omega_{N}$ describe transitions induced by quark colour singlet currents (19)-(20) in the first, second and third terms of eq. (12). Diagrams in fig.1,2 with the intermediate states $\{W-N-$ $W\},\{\tilde{u}(\tilde{d})-\chi, \tilde{g}-\tilde{u}(\tilde{d})\}$ and $\{\tilde{u}(\tilde{d})-\chi, \tilde{g}-\tilde{d}(\tilde{u}) ; \tilde{e}-\chi-\tilde{e}, \tilde{q}\}$ contribute to operators $\Omega_{N}, \Omega_{\tilde{q}}$ and $\Omega_{\tilde{f}}$ respectively. Calculation of the transition operators in the non-relativistic impulse approximation (NRIA) [2] requires the nucleon matrix elements of these currents.

We derive nucleon matrix elements of the colour singlet quark currents (19)-(20) using results of ref. [22]. The necessary matrix elements are,

$$
<P(p)|\bar{u} d| N\left(p^{\prime}\right)>=F_{S}^{(3)}\left(q^{2}\right) \cdot \bar{N}(p) \tau_{+} N\left(p^{\prime}\right),
$$


Table 1: Nucleon form factor normalizations at $q^{2}=0$ as calculated in [22].

\begin{tabular}{|c|l|l|l|l|l|}
\hline Set & $F_{S}^{(3)}$ & $F_{P}^{(3)}$ & $T_{1}^{(3)}$ & $T_{2}^{(3)}$ & $T_{3}^{(3)}$ \\
\hline A) Bag Model & 0.48 & 4.41 & 1.38 & -3.30 & -0.62 \\
\hline $\begin{array}{c}\text { B) Non-relativistic } \\
\text { quark model }\end{array}$ & 0.62 & 4.65 & 1.45 & -1.48 & -0.66 \\
\hline
\end{tabular}

$$
\begin{aligned}
<P(p)\left|\bar{u} \gamma_{5} d\right| N\left(p^{\prime}\right)> & =F_{P}^{(3)}\left(q^{2}\right) \cdot \bar{N}(p) \gamma_{5} \tau_{+} N\left(p^{\prime}\right) \\
<P(p)\left|\bar{u} \sigma^{\mu \nu}\left(1+\gamma_{5}\right) d\right| N\left(p^{\prime}\right)> & =\bar{N}(p)\left(J^{\mu \nu}+\frac{i}{2} \epsilon^{\mu \nu \rho \sigma} J_{\rho \sigma}\right) \tau_{+} N\left(p^{\prime}\right) \\
<P(p)\left|\bar{u} \gamma^{\mu}\left(1-\gamma_{5}\right) d\right| N\left(p^{\prime}\right)> & =\bar{N}(p) \gamma^{\mu}\left(F_{V}\left(q^{2}\right)-F_{A}\left(q^{2}\right) \gamma_{5}\right) \tau_{+} N\left(p^{\prime}(2)\right.
\end{aligned}
$$

where $q=p-p^{\prime}$ and the tensor structure is defined as

$$
J^{\mu \nu}=T_{1}^{(3)}\left(q^{2}\right) \sigma^{\mu \nu}+\frac{i T_{2}^{(3)}}{m_{P}}\left(\gamma^{\mu} q^{\nu}-\gamma^{\nu} q^{\mu}\right)+\frac{T_{3}^{(3)}}{m_{P}^{2}}\left(\sigma^{\mu \rho} q_{\rho} q^{\nu}-\sigma^{\nu \rho} q_{\rho} q^{\mu}\right) .
$$

For all form factors $F_{V, A}\left(q^{2}\right), F_{S, P}^{(3)}\left(q^{2}\right), T_{i}^{(3)}\left(q^{2}\right)$ we take, following ref. [23], a dipole form

$$
\frac{F_{V, A}\left(q^{2}\right)}{f_{V, A}}=\frac{F_{S, P}^{(3)}\left(q^{2}\right)}{F_{S, P}^{(3)}(0)}=\frac{T_{i}^{(3)}\left(q^{2}\right)}{T_{i}^{(3)}(0)}=\left(1-\frac{q^{2}}{m_{A}^{2}}\right)^{-2}
$$

with $m_{A}=0.85 \mathrm{GeV}$ and $f_{V} \approx 1, f_{A} \approx 1.261$. Form factor normalization values $F_{S, P}^{(3)}(0), T_{i}^{(3)}(0)$ were calculated in ref. [22] and are given in Table 1.

Using formulas (22)-(26) we may derive the non-relativistic limit $m_{P} \gg|\vec{p}|$ for nucleon matrix elements of the three combinations of the quark currents in (12). Keeping all terms up to order $q^{2}$ in the non-relativistic expansion we find the relevant transition operators $\Omega_{\tilde{q}}, \Omega_{\tilde{f}}, \Omega_{N}$ for the case of two outgoing electrons in S-wave states,

$$
\begin{aligned}
\Omega_{\tilde{q}} & =\frac{m_{P}}{m_{e}}\left\{\alpha_{V}^{(0)} \Omega_{F, N}+\alpha_{A}^{(0)} \Omega_{G T, N}+\alpha_{V}^{(1)} \Omega_{F^{\prime}}+\alpha_{A}^{(1)} \Omega_{G T^{\prime}}+\alpha_{T} \Omega_{T^{\prime}}\right\}, \\
\Omega_{\tilde{f}} & =\Omega_{\tilde{q}}\left(T_{i}=0\right), \\
\Omega_{N} & =\frac{m_{P}}{m_{e}}\left\{\left(\frac{f_{V}}{f_{A}}\right)^{2} \Omega_{F, N}-\Omega_{G T, N}\right\},
\end{aligned}
$$

where partial transition operators are

$$
\Omega_{G T, N}=\sum_{i \neq j} \tau_{+}^{(i)} \tau_{+}^{(j)} \sigma_{i} \cdot \sigma_{j}\left(\frac{R_{0}}{r_{i j}}\right) F_{N}\left(x_{A}\right),
$$




$$
\begin{aligned}
\Omega_{F, N} & =\sum_{i \neq j} \tau_{+}^{(i)} \tau_{+}^{(j)}\left(\frac{R_{0}}{r_{i j}}\right) F_{N}\left(x_{A}\right), \\
\Omega_{G T^{\prime}} & =\sum_{i \neq j} \tau_{+}^{(i)} \tau_{+}^{(j)} \sigma_{i} \cdot \sigma_{j}\left(\frac{R_{0}}{r_{i j}}\right) F_{4}\left(x_{A}\right), \\
\Omega_{F^{\prime}} & =\sum_{i \neq j} \tau_{+}^{(i)} \tau_{+}^{(j)}\left(\frac{R_{0}}{r_{i j}}\right) F_{4}\left(x_{A}\right), \\
\Omega_{T^{\prime}} & =\sum_{i \neq j} \tau_{+}^{(i)} \tau_{+}^{(j)}\left\{3\left(\sigma_{i} \cdot \hat{r}_{i j}\right)\left(\sigma_{j} \cdot \hat{r}_{i j}\right)-\sigma_{i} \cdot \sigma_{j}\right\}\left(\frac{R_{0}}{r_{i j}}\right) F_{5}\left(x_{A}\right) .
\end{aligned}
$$

Here, $R_{0}$ is the nuclear radius, introduced to make the matrix elements dimensionless (compensating factors have been absorbed into the phase space integrals [2]). The following notations are used $r_{i j}=\left(\vec{r}_{i}-\vec{r}_{j}\right), r_{i j}=\left|r_{i j}\right|, \hat{r}_{i j}=$ $\boldsymbol{r}_{i j} / r_{i j}, x_{A}=m_{A} r_{i j}$. We also define the normalization constant in eq. (21) $C_{0 \nu}=4 \pi \frac{m_{P}}{m_{e}} \frac{R_{0}}{f_{A}^{2}} m_{A}^{-2}$. The above formulae have been written in the closure approximation, which is well satisfied for large masses of intermediate particles [2].

The nucleon structure coefficients in (28) are given by

$$
\begin{aligned}
& \alpha_{V}^{(0)}=\left(\frac{F_{S}^{(3)}}{f_{A}}\right)^{2}, \alpha_{A}^{(0)}=-\left(\frac{T_{1}^{(3)}}{f_{A}}\right)^{2} \\
& \alpha_{V}^{(1)}=-\left(\frac{m_{A}}{m_{P}}\right)^{2} \alpha_{A}^{(0)}\left[\frac{1}{4}+\left(\frac{T_{2}^{(3)}}{T_{1}^{(3)}}\right)^{2}-\frac{T_{2}^{(3)}}{T_{1}^{(3)}}\right] \\
& \alpha_{A}^{(1)}=-\left(\frac{m_{A}}{m_{P}}\right)^{2}\left[\alpha_{A}^{(0)}\left(\frac{1}{6}-\frac{2}{3} \frac{T_{2}^{(3)}}{T_{1}^{(3)}}+\frac{4}{3} \frac{T_{3}^{(3)}}{T_{1}^{(3)}}\right)+\frac{1}{12}\left(\frac{F_{P}^{(3)}}{f_{A}}\right)^{2}\right] \\
& \alpha_{T}=-\left(\frac{m_{A}}{m_{P}}\right)^{2}\left[\alpha_{A}^{(0)}\left(\frac{1}{12}-\frac{1}{3} \frac{T_{2}^{(3)}}{T_{1}^{(3)}}+\frac{2}{3} \frac{T_{3}^{(3)}}{T_{1}^{(3)}}\right)-\frac{1}{12}\left(\frac{F_{P}^{(3)}}{f_{A}}\right)^{2}\right] .
\end{aligned}
$$

Here $F_{S, P}^{(3)} \equiv F_{S, P}^{(3)}(0), T_{i}^{(3)} \equiv T_{i}^{(3)}(0)$. Three different structure functions $F_{i}$ appear in eq. (28) (we use notations of ref. [21]). They are given by

$$
\begin{gathered}
F_{N}(x)=\frac{x}{48}\left(3+3 x+x^{2}\right) e^{-x}, \quad F_{4}(x)=\frac{x}{48}\left(3+3 x-x^{2}\right) e^{-x}, \\
F_{5}(x)=\frac{x^{3}}{48} e^{-x} .
\end{gathered}
$$


These functions are the analytic solutions of the relevant integrals over the intermediate particle momentum. They are analogons to the "neutrino potentials" for the case of light Majorana neutrino exchange [2].

At this stage we point out that our formulas for the coefficients (36)-(39) of the transition operators (28)-(29) disagree with the corresponding formulas derived in ref. [21]. Particularly, in our case $\alpha_{A}^{(0)} \leq 0$ while in ref. [21] this coefficient is positive. This sign difference has an important consequence if one considers simultaneously the supersymmetric and the heavy Majorana neutrino contributions. Our formulas correspond to a constructive interference between these two mechanisms while formulas from ref. [21] correspond to a destructive one. In the latter case both contributions can cancel each other and by a proper choice of $\lambda_{111}^{\prime}$ in eqs. (13)-(17) the matrix element of $0 \nu \beta \beta$-decay can be set to zero at any values of particle masses involved in the formulas. As a result the $0 \nu \beta \beta$-decay half-life limit would neither constrain supersymmetric particle masses nor that for Majorana neutrinos. Our formulas (28)-(39) always lead to certain constraints on these masses. Detailed discussion of this point will be given elsewhere.

In conclusion we note that the results obtained in the present paper can be directly used for the extraction of constraints on the supersymmetric parameter space from the non-observation of $0 \nu \beta \beta$ decay. To do this one should employ nuclear wave functions calculated in a specific nuclear structure model. A subsequent paper will be devoted to this subject.

\section{ACKNOWLEDGMENTS}

We thank V.A. Bednyakov, V.B. Brudanin, M. Lindner and J.W.F. Valle for helpful discussions. The research described in this publication was made possible in part by Grant No.RFM000 from the international Science Foundation.

\section{References}

[1] W.C. Haxton and G.J. Stephenson, Progr. Part. Nucl. Phys. 12 (1984) 409 K. Grotz and H.V. Klapdor-Kleingrothaus, The Weak Interactions in Nuclear, Particle and Astrophysics, Adam Hilger, Bristol, New York, 1990; M. Moe and P. Vogel, Annual Review of Nucl. and Part. Science 44 (1994) 247 
[2] M. Doi, T. Kotani and E. Takasugi, Progr. Theor. Phys. Suppl. 83 (1985) 1; J. D. Vergados, Phys. Report, 133 (1986) 1; J.W.F. Valle, Prog. Part. Nucl. Phys. 26 (1991) 91.

[3] HEIDELBERG-MOSCOW Collaboration: A. Balysh et al., Proceedings of the 27th Int. Conf. on High Energy Physics, 20th-27th July 1994, Glasgow in the press and manuscript submitted to Phys.Lett. B;

H.V.Klapdor-Kleingrothaus, Progr.Part.Nucl.Phys. 32 (1994) p.261

[4] H.E. Haber and G.L.Kane, Phys.Rep. 117 (1985) 75; H.P. Nilles, Phys.Report. 110 (1984) 1.

[5] S. Dimopoulos and L.J. Hall, Phys.Lett. B207 (1988) 210. E. Ma and D. Ng, Phys. Rew. D41 (1990) 1005,

[6] L. Hall and M. Suzuki, Nucl.Phys. B 231 (1984) 419.

[7] C. Aulakh and R. Mohapatra, Phys.Lett. B119 (1983) 136; G.G. Ross and J.W.F. Valle, Phys.Lett. B151 (1985) 375; J. Ellis et al., Phys.Lett. B150 (1985) 142; A. Santamaria and J.W.F. Valle, Phys.Lett. B195 (1987) 423; Phys.Rev.Lett. 60 (1988) 397; Phys.Rev. D 39 (1987) 1780; M.C. Gonzalez-Garsia and J.W.F. Valle, Nucl.Phys. B355 (1991) 330; J.W.F. Valle, Phys.Lett. B196 (1987) 157;

[8] A. Masiero and J.W.F. Valle Phys.Lett. B251 (1990) 273.

[9] V. Barger, G.F. Guidice and T. Han, Phys. Rev. D 40 (1989) 2987; R. Barbieri and A. Masiero, Nucl.Phys. B267 (1986) 679; D.P. Roy, Phys. Lett. B 283 (1992) 270;

[10] F. Zwirner, Phys. Lett. B132 (1983) 103.

[11] S. Weinberg, Phys.Rev. D26 (1982) 287;

[12] A. Bouquet and P. Salati, Nucl.Phys. B284 (1987); A. Nelson and S.M. Barr, Phys.Lett. B258 (1991) 45;

[13] B.A. Campbell, S. Davidson, J. Ellis and K. Olive, Phys.Lett. B256 (1991) 457; CERN-preprint, CERN-TH-6208-91; W. Fieschler, G. Guidice, R.G. Leigh and S. Paban, Phys.Lett. B258 (1991) 45. 
[14] S. Dimopoulos and L.J. Hall, Phys.Lett. B196 (1987) 135; R. Mohapatra and J.W.F. Valle, Phys.Lett. B186 (1987) 303; J. Cline S. Rabby Ohio State preprint,1990.

[15] H. Dreiner and G. Ross, Nucl. Phys. B 410 (1994) 188.

[16] S. Dimopoulos, S. Raby and F. Wilszek, Phys.Lett. B112 (1982) 133; N. Sakai and T. Yanagida, Nucl.Phys. B197 (1982) 83;

[17] D. Braham and L. Hall, Phys.Rev. D40 (1989) 2449;

[18] M. Bento, L.J. Hall and G.G. Ross, Nucl.Phys. B292 (1987) 400; G. Lazarides, P.K. Mohapatra, C. Panagiotakopoulos and Q. Shafi, Nucl.Phys. B323 (1989) 614;

[19] J.F. Gunion, H.E. Haber, G.L. Kane, Nucl.Phys. B 272 (1986) 1.

[20] R. Mohapatra, Phys.Rev. D34 (1986) 3457.

[21] J.D Vergados, Phys.Lett. B184 (1987) 55;

[22] S.L. Adler et al., Phys.Rev. D11 (1975) 3309;

[23] J.D. Vergados, Phys.Rev. C24 (1981) 640; J.D. Vergados, Nucl.Phys. B218 (1983) 109.

\section{Figure Captions}

Fig.1 Feynman graphs for the conventional mechanism of $0 \nu \beta \beta$ decay by massive Majorana neutrino exchange.

Fig.2a Feynman graphs for supersymmetric contributions to $0 \nu \beta \beta$ decay.

Fig.2b New "non-diagonal" Feynman graphs for supersymmetric contributions to $0 \nu \beta \beta$ decay. 
Figure 1.

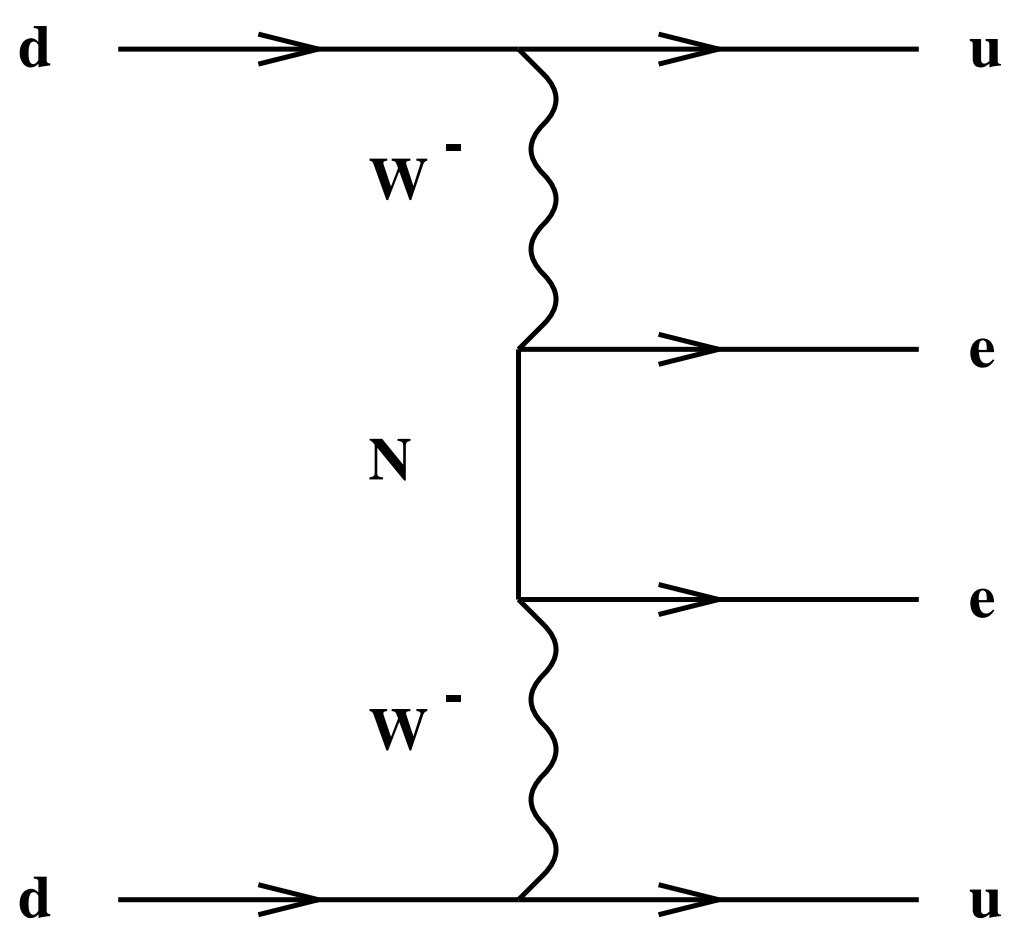


Figure 2.a
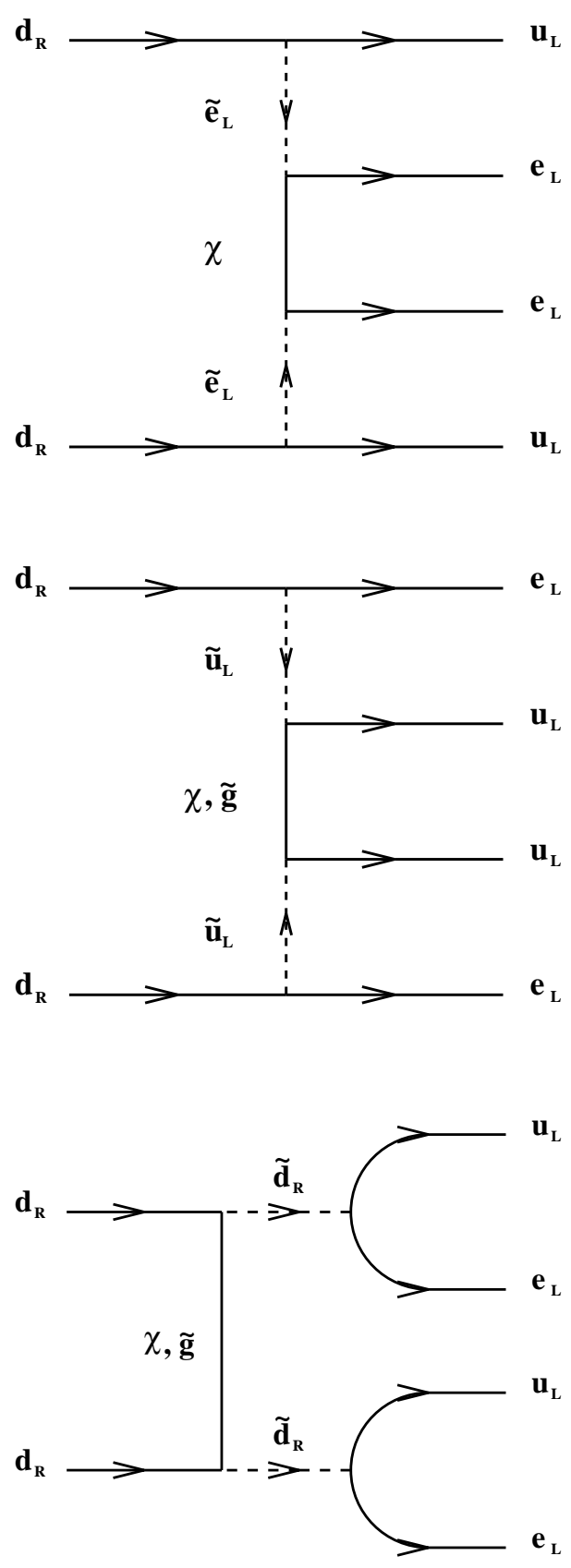
Figure 2.b
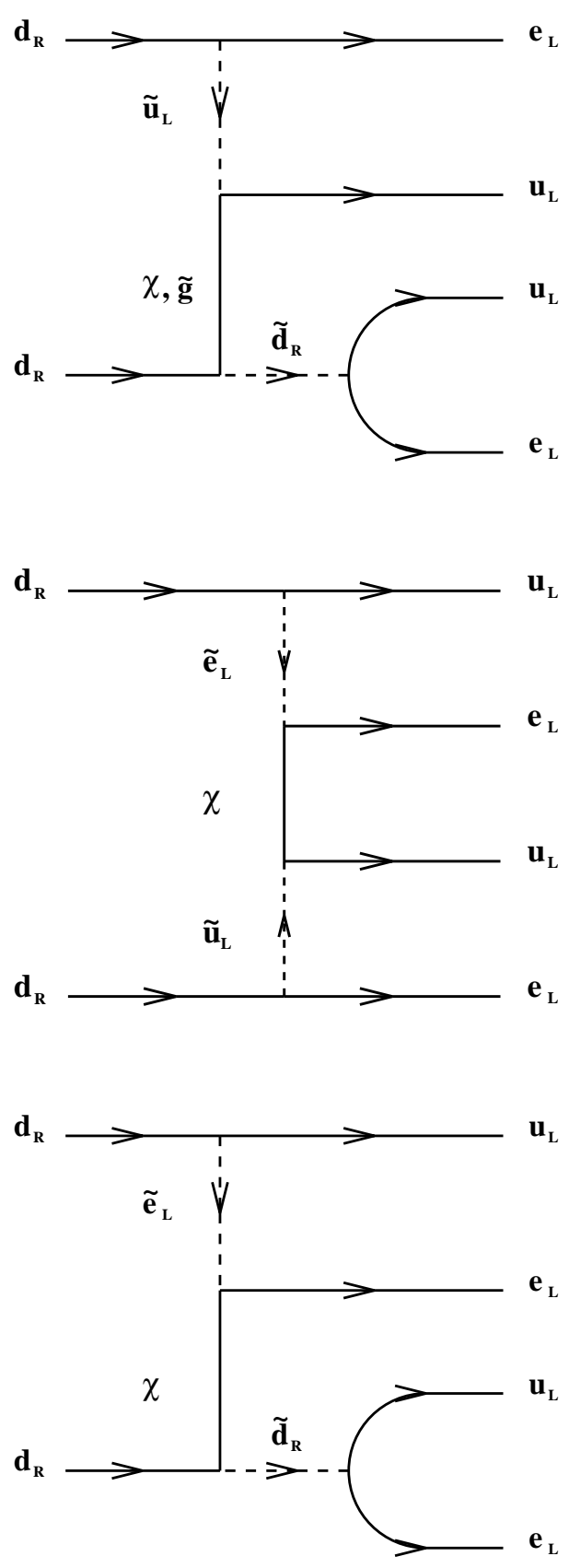